\title{
Multi-class brain tumor segmentation via multi-sequences MRI mixture data preprocessing
}

\author{
Andrey Letyagin \\ Research Institute of Clinical and \\ Experimental \\ Lymphology, Branch of IC\&G SB RAS \\ Novosibirsk, Russia \\ letyaginay@bionet.nsc.ru \\ Sergey Golushko \\ Novosibirsk State University \\ Novosibirsk, Russia \\ s.k.golushko@gmail.com \\ Mikhail Amelin \\ Radiodiagnosis division \\ FSBI "Federal Neurosurgical Center" \\ Novosibirsk, Russia \\ amelin81@gmail.com
}

\author{
Bair Tuchinov \\ SDAML lab. \\ Novosibirsk State University \\ Novosibirsk, Russia \\ bairts@gmail.com \\ Evgeniya Amelina \\ SDAML lab. \\ Novosibirsk State University \\ Novosibirsk, Russia \\ amelina.evgenia@gmail.com \\ Nikolay Tolstokulakov \\ SDAML lab. \\ Novosibirsk State University \\ Novosibirsk, Russia \\ n.tolstokulakov@g.nsu.ru
}

\author{
Evgeniy Pavlovskiy \\ SDAML lab. \\ Novosibirsk State University \\ Novosibirsk, Russia \\ pavlovskiy@post.nsu.ru \\ Vladimir Groza \\ Median Technologies \\ Valbonne, France \\ vladimir.groza@gmail.com
}

\begin{abstract}
In this paper, we extend the previous work on the robust pre-processing technique which allows to consider all available information from MRI scans by composition of T1, T1C and FLAIR sequences in the unique input. Such approach enriches the input data for the automatic segmentation process and helps to improve the accuracy of the segmentation performance.
\end{abstract}

Keywords - Neural Network, Deep Learning, Semantic segmentation, Medical Imaging

Proposed method also demonstrates significant improvement on the multiclass segmentation problem with respect to Dice metrics compare to similar training / evaluation procedure based on any single sequence regardless of the chosen neural network architecture.

Obtained results demonstrates significant evaluation improvement while combining three MRI sequences in the 3-channel RGB like image for considered problem of multiclass brain tumor segmentation. We also provide results of comparison of various gradient descent optimization methods and of different backbone architectures. We found that different algorithms worked best for different tumors, but no single algorithm ranked in the top for all types of tumors simultaneously. Final improvements on the test part of our dataset are in the range of $6-9 \%$ on the trained model according to the Dice metric with the best value of 0.949 .

\section{ACKNOWLEDGMENT}

The reported study was funded by RFBR according to the research project No. 19-29-01103.

\section{REFERENCES}

[1] Kamnitsas, K., Bai, W., Ferrante, E., McDonagh, S., Sinclair, M., et al. Ensembles of Multiple Models and Architectures for Robust Brain Tumour Segmentation. MICCAI Brainlesion Workshop. pp. 450-462. Springer, 2017.

[2] Myronenko, A. 3D MRI Brain Tumor Segmentation using Autoencoder Regularization. MICCAI Brainlesion Workshop. pp. 311-320. Springer, 2018.

[3] Letyagin, A. Y., Golushko, S. K., Rzaev, J. A., Amelin, M. E.,

[4] Pavlovskiy, E. N., Tuchinov, B. N., ... Degtyareva, L. O. Artificial Intelligence for Imaging Diagnostics in Neurosurgery. In 2019 International Multi-Conference on Engineering, Computer and Information Sciences (SIBIRCON) (pp. 336-337). IEEE-INST ELECTRICAL ELECTRONICS ENGINEERS INC.

[5] Vladimir Groza, Bair Tuchinov, Evgeniy Pavlovskiy, Evgeniya Amelina, Mihail Amelin, Sergey Golushko, Andrey Letyagin. Data Preprocessing via Multi-sequences MRI Mixture to Improve Brain Tumor Segmentation Bioinformatics and Biomedical Engineering, 2020 (pp. 695-704). Springer, 2020. 